\title{
Effervescent heating: constraints from nearby cooling flow clusters observed with XMM-Newton
}

\author{
R. Piffaretti ${ }^{1}$ and J. S. Kaastra ${ }^{2}$ \\ 1 Institut für Astrophysik, Leopold-Franzens Universität Innsbruck, Technikerstraße 25, 6020 Innsbruck, Austria \\ e-mail: Rocco.Piffaretti@uibk.ac.at \\ 2 SRON National Institute for Space Research, Sorbonnelaan 2, 3584 CA Utrecht, The Netherlands
}

Received 17 October 2005 / Accepted 31 January 2006

ABSTRACT

\begin{abstract}
Aims. We have used deprojected radial density and temperature profiles of a sample of 16 nearby CF clusters observed with $X M M$-Newton to test whether the effervescent heating model can satisfactorily explain the dynamics of CF clusters.

Methods. For each cluster we derived the required extra heating as a function of cluster-centric distance for various values of the unknown parameters $\dot{M}$ (mass deposition rate) and $f_{\mathrm{c}}$ (conduction efficiency). We fitted the extra heating curve using the AGN effervescent heating function and derived the AGN parameters $L$ (the time-averaged luminosity) and $r_{0}$ (the scale radius where the bubbles start rising in the ICM).

Results. While we do not find any solution with the effervescent heating model for only one object, we do show that AGN and conduction heating are not cooperating effectively for half of the objects in our sample. For most of the clusters we find that, when a comparison is possible, the derived AGN scale radius $r_{0}$ and the observed AGN jet extension have the same order of magnitude. The AGN luminosities required to balance radiative losses are substantially lowered if the fact that the AGN deposits energy within a finite volume is taken into account. For the Virgo cluster, we find that the AGN power derived from the effervescent heating model is in good agreement with the observed jet power.
\end{abstract}

Key words. X-rays: galaxies: clusters - cooling flows - conduction - galaxies: active

\section{Introduction}

The radiative cooling time of the intracluster medium (ICM) in the cores of many galaxy clusters is short enough for the plasma to radiate an amount of energy equal to its thermal energy in less than a billion years. Therefore the gas should gradually condense, be replaced by the surrounding material, and ultimately form a cooling flow (hereafter $\mathrm{CF}$ ). Early estimates of the mass deposition rates from X-ray imaging $\left(\sim 10^{2}-10^{3} M_{\odot} \mathrm{yr}^{-1}\right)$ have indicated that the cores of CF clusters should contain large amounts of cold gas, but searches for these large amounts of condensed baryons have not been successful (see Fabian 1994 for a review of the subject of CFs before the launch of the XMM-Newton and Chandra satellites).

XMM-Newton and Chandra observations have greatly improved our understanding of CF clusters. Most important, X-ray spectra failed to detect the emission lines that dominate the emission from gas below $2 \mathrm{keV}$ (Peterson et al. 2001; Tamura et al. 2001; Kaastra et al. 2001; Peterson et al. 2003), therefore ruling out the standard CF model. In addition, as shown by Peterson et al. (2003), the observed spectra cannot be explained without any substantial modifications to the general CF process. Recent observations have also shown that mass deposition rates are significantly smaller than those estimated previously (Böhringer et al. 2001; David et al. 2001; Peterson et al. 2003). Even though this implies a less severe discrepancy between CF mass deposition and star-formation rates (McNamara 2004), no agreement between the amount of cold baryons predicted from X-ray observations and the one observed in other wavelengths has been found yet. Spatially-resolved spectroscopy shows that the temperature in CF clusters drops towards the center to approximatively one third of the cluster mean temperature, indicating that the gas is prevented from cooling below these cutoff temperatures (Peterson et al. 2001; Tamura et al. 2001; Kaastra et al. 2004).

This recent evidence shows that the dynamics of the ICM in CF clusters is not solely governed by cooling of the ICM, and that some heating and/or non X-ray cooling mechanisms must be investigated. While non X-ray, rapid cooling mechanisms have been studied (see Peterson et al. 2003, and references therein), the most appealing mechanisms involve heating processes.

Most CF clusters host a central active galactic nucleus (AGN) with strong radio activity (Burns 1990; Ball et al. 1993) and, most important, recent observations show that these radio sources are interacting with the ICM and often displace the hot gas, leaving cavities in their wakes (e.g., see Blanton 2004; Bîrzan et al. 2004, and references therein). Hence, it has been realized that gas heating by the outflows of the central AGN can be a vital process for the dynamics of CFs. This heating mechanism involves buoyant plasma bubbles inflated by the AGN, which then rise through the cluster atmosphere, expand, and ultimately heat the ICM (Churazov et al. 2002; Brüggen \& Kaiser 2001; Ruszkowski \& Begelman 2002; Brüggen \& Kaiser 2002). Although there is no consensus regarding the ability of AGN heating to prevent the formation of CFs and the efficiency of the processes by which energy is delivered to the ICM, there is evidence suggesting that the class of models in which the AGN energy input alone balances radiative losses is unable to quench the CF (Brighenti \& Mathews 2002; Zakamska \& Narayan 2003). 
Table 1. The radius at the center of the last radial bin considered in the temperature fits and the best-fit parameters from fitting Eqs. (1) and (2) to the data ( $1 \sigma$ errors in parenthesis). Here, $\mu=2$ in Eq. (1) is used and, for both fitting functions, $T(r=0)$ is set equal to the temperature of the central bin.

\begin{tabular}{l|c|ccc|ccc}
\hline \hline Cluster & $\begin{array}{c}R_{\mathrm{T}, \mathrm{max}} \\
(\mathrm{kpc})\end{array}$ & $\begin{array}{c}T_{0} \\
(\mathrm{keV})\end{array}$ & $\begin{array}{c}T_{1} \\
(\mathrm{keV})\end{array}$ & $\begin{array}{c}r_{\mathrm{T}} \\
(\mathrm{kpc})\end{array}$ & $\begin{array}{c}\tilde{T}_{0} \\
(\mathrm{keV})\end{array}$ & $\begin{array}{c}\tilde{T}_{1} \\
(\mathrm{keV})\end{array}$ & $\begin{array}{c}\tilde{r}_{\mathrm{T}} \\
(\mathrm{kpc})\end{array}$ \\
\hline NGC 533 & 75 & $0.67(0.01)$ & $0.81(0.03)$ & $18(1)$ & $1.35(0.17)$ & $0.68(0.16)$ & $17(4)$ \\
Virgo & 35 & $1.45(0.02)$ & $1.27(0.18)$ & $16(3)$ & $2.45(0.13)$ & $1.00(0.11)$ & $11(2)$ \\
A262 & 199 & $1.02(0.02)$ & $1.24(0.08)$ & $21(2)$ & $2.16(0.08)$ & $1.14(0.05)$ & $16(1)$ \\
Sérsic 159-3 & 233 & $2.17(0.05)$ & $0.29(0.04)$ & $38(12)$ & $2.42(0.07)$ & $0.25(0.02)$ & $30(5)$ \\
MKW 9 & 167 & $1.28(0.09)$ & $1.40(0.31)$ & $57(16)$ & $2.42(0.24)$ & $1.14(0.15)$ & $40(6)$ \\
2A 0335+096 & 206 & $1.40(0.03)$ & $1.76(0.16)$ & $52(6)$ & $2.88(0.14)$ & $1.48(0.11)$ & $38(3)$ \\
MKW 3s & 268 & $3.00(0.10)$ & $0.57(0.12)$ & $40(20)$ & $3.52(0.21)$ & $0.52(0.10)$ & $36(14)$ \\
A2052 & 212 & $1.41(0.06)$ & $1.79(0.14)$ & $27(5)$ & $3.03(0.15)$ & $1.62(0.09)$ & $22(2)$ \\
A4059 & 275 & $2.11(0.13)$ & $2.17(0.15)$ & $44(7)$ & $4.03(0.23)$ & $1.92(0.09)$ & $35(3)$ \\
Hydra A & 321 & $2.92(0.10)$ & $0.63(0.12)$ & $114(30)$ & $3.46(0.19)$ & $0.54(0.08)$ & $88(16)$ \\
A496 & 289 & $2.14(0.10)$ & $2.35(0.20)$ & $59(7)$ & $4.20(0.22)$ & $2.06(0.12)$ & $45(3)$ \\
A3112 & 645 & $2.99(0.08)$ & $1.55(0.21)$ & $61(17)$ & $4.36(0.20)$ & $1.37(0.12)$ & $46(8)$ \\
A1795 & 369 & $3.47(0.09)$ & $2.67(0.20)$ & $90(9)$ & $5.73(0.19)$ & $2.26(0.10)$ & $65(4)$ \\
A399 & 404 & $2.60(0.57)$ & $4.29(0.44)$ & $67(17)$ & $6.61(0.91)$ & $4.01(0.34)$ & $58(11)$ \\
Perseus & 229 & $3.07(0.05)$ & $3.91(1.10)$ & $132(28)$ & $5.76(0.58)$ & $2.69(0.53)$ & $79(11)$ \\
A1835 & 594 & $5.13(0.14)$ & $2.78(0.24)$ & $207(68)$ & $7.34(0.77)$ & $2.21(0.63)$ & $134(50)$ \\
\hline \multicolumn{7}{c}{}
\end{tabular}

Despite the fact that the presence of magnetic fields in clusters implies that thermal conductivity is only a fraction of the Spitzer value, thermal conduction by electrons might play an important role in CFs. While some theoretical work has pointed out that thermal conduction must be highly suppressed (e.g., see Fabian 1994), recent theoretical papers show that conductivity can be as high as a substantial fraction of the Spitzer rate (Narayan \& Medvedev 2001; Gruzinov 2002) and therefore it has been reconsidered as a possible heat source candidate. However, it has been shown that heat conduction alone fails to balance radiative losses at the center of the clusters (Voigt et al. 2002; Zakamska \& Narayan 2003; Voigt \& Fabian 2004; Kaastra et al. 2004) and that models with heat conduction as the only heating source are unstable (Soker 2003).

Other, less popular heating mechanisms are: fluid turbulence generated by substructure motion or cluster mergers (Fujita et al. 2004), heating from intra-cluster supernovae (Domainko et al. 2004), heating by hadronic cosmic rays (Colafrancesco et al. 2004), and heating by magnetic fields (Soker \& Sarazin 1990; Makishima et al. 2001).

The inability of heat conduction to balance radiative losses and the failure of some AGN heating models have motivated the development of models in which these two mechanisms cooperate. Models with heat conduction and AGN heating acting together are very attractive due to the complementary nature of the two processes: thermal conduction is effective/ineffective in the outer/inner regions of the cluster, and AGN heating is effective/ineffective in the inner/outer part. Ruszkowski \& Begelman (2002) (hereafter RB02) have recently proposed such a model. The ICM density and temperature evolved according to their model reach a final stable configuration in agreement with the general shape of observed density and temperature profiles in CF clusters. RB02's model (or the effervescent heating model, hereafter) is the only proposed theoretical model that can be effectively tested against observations. Unfortunately this has only been done for the Virgo cluster (Ghizzardi et al. 2004), and it is not clear whether the model can give a satisfactory explanation of the dynamics of CF clusters in general.

In the present work we address the latter question using a sample of CF clusters observed with XMM-Newton. Our sample, which consists of 16 objects, is large for this kind of analysis and all the objects were analyzed using an appropriate and homogeneous procedure.

The layout of this paper is as follows. In Sect. 2 we present the sample and briefly summarize the procedure used to derive the basic quantities used in our analysis. In Sect. 3 we give a description of RB02's model and present the equations used in Sect. 4, where we fit the model to the data. In Sect. 5 we summarize the results of our analysis and present the conclusions.

We adopt $H_{0}=70 \mathrm{~km} \mathrm{~s}^{-1} \mathrm{Mpc}^{-1}, \Omega_{\mathrm{m}}=0.3$ and $\Omega_{\Lambda}=0.7$ in all the calculations throughout this paper.

\section{Data}

The sample consists of the clusters listed in Table 1. These objects are part of the sample analyzed in Kaastra et al. (2004; hereafter K04), who derived deprojected radial temperature and density profiles for all the clusters in the sample, including a description of the sample and an extensive presentation of the data analysis. From the K04's sample, we excluded 4 clusters: Coma, A754 and A3266, because these are non-CF clusters, and A1837 since temperature and density could be measured in only one bin within the cooling radius. Spectral fitting was done over the full 0.2-10 keV range and, in general, temperatures and gas densities were computed for the innermost 8 shells (see K04, Table 3 for the boundaries between the shells), except when the data became too noisy in the outermost shell. The sample used in the present work therefore consists of 16 clusters with CF for which gas density and temperature radial profiles are well measured. Since thermal conduction depends on temperature gradients and the AGN heating in the RB02 model involves a dependency on ICM pressure, we model gas temperature and density profiles by fitting the data with analytic functions. We used more that one fitting function for both quantities in order to quantify the difference due to the modelling. In the following we describe these functions and the fitting to the data.

\subsection{Modelling of gas temperature and density}

The deprojected temperature profiles of our clusters exhibit a self-similar shape studied in Piffaretti et al. (2005): the temperature declines from the maximum cluster temperature at a break 
radius $r_{\mathrm{br}}$ moving outwards and shows the characteristic temperature decline towards the X-ray emission peak. Since we are interested in the central cooling region and the cooling radius for a cooling time of $15 \mathrm{Gyr}, R_{\mathrm{cool}}$ is smaller than $r_{\mathrm{br}}$ for all the clusters, the temperature profiles can be simply modelled by a function that is monotonically raising with radius. Hence, for each cluster we select temperature bins inside the radius $R_{\mathrm{T}, \max }=r_{\mathrm{br}}$ and fit them using the following expressions:

$T(r)=T_{0}+T_{1} \frac{\left(r / r_{\mathrm{T}}\right)^{\mu}}{1+\left(r / r_{\mathrm{T}}\right)^{\mu}}$,

or

$T(r)=\tilde{T}_{0}-\tilde{T}_{1} \exp \left(-\frac{r^{2}}{2 \tilde{r}_{\mathrm{T}}^{2}}\right)$.

In order to reduce the number of parameters here, we set $T(r=0)$ equal to the temperature of the central bin for both fits and use $\mu=2$ in Eq. (1) (Allen et al. 2001). For Perseus, Virgo, A262, A496, and A3112, the temperature in the observed radial range is monotonically raising with radius, and therefore $R_{\mathrm{T} \text {,max }}$ is just the distance of the outermost bin from the cluster center. The best-fit parameters and $R_{\mathrm{T}, \max }$ are given in Table 1 . Both temperature parametrizations are used in the computation of thermal conduction and in the modelling of the gas pressure. Our main results presented in Sect. 4 are achieved using the parametrization in Eq. (1), and we use the second parametrization given in Eq. (2) only in order to explore the effect of a different modelling on our main results. The changes introduced by using Eq. (2) are, as we will show in Sect. 4.4, quite small and do not change the results obtained by using Eq. (1).

We model the gas density by using a single $\beta$-model given by:

$\rho(r)=\rho_{0}\left(1+\left(r / r_{\mathrm{c}}\right)^{2}\right)^{-\frac{3}{2} \beta}$.

The density profile is fitted within $R_{\rho, \max }$, which is the radius at the center of the last radial bin, where a robust estimate of gas density and temperature is possible (see Piffaretti et al. 2005 for the bin selection criterion). The best-fit parameters for the single $\beta$-model and $R_{\rho \text {,max }}$ are given in Table 2 .

An alternative parametrization of the gas density profile is the more complex double $\beta$-model, which is a popular generalization of the single $\beta$-model used to model the central surfacebrightness excess observed in CF clusters. Unfortunately in this case, the gas density is modelled using the sum of two single $\beta$-models, so the number of free parameters is doubled: $\rho_{0, i}, \beta_{i}$, and $r_{\mathrm{c}, i}$, with $i=1,2$. As a consequence, while fitting the single $\beta$-model to the density profiles gives statistically significant values for the best-fit parameters, the large number of parameters adopted in the double $\beta$-model, together with the small number of bins in which the gas density is measured, do not allow a significant determination of the best-fit parameters. Therefore, we decided to present our main results in Sect. 4 using the single $\beta$-model. Nonetheless, in order to constrain to which extent the double $\beta$-modelling changes the results, we also fit the density profiles using a double $\beta$-model with a reduced number of fitting parameters, by setting $\beta_{1}=\beta_{2}$ and fixing one of the core radii equal to the core radius $r_{\mathrm{T}}$ (the core radius $\tilde{r}_{\mathrm{T}}$ is also used as a supplementary choice). The derived profiles are then used to model the gas pressure as done by using the single $\beta$-model results. A discussion of the influence of this different modelling on our main results is given in Sect. 4.4 below.
Table 2. The radius at the center of the last radial bin considered in the gas density fits and the best-fit parameters from fitting Eq. (3) to the data ( $1 \sigma$ errors in parenthesis).

\begin{tabular}{l|c|ccc}
\hline \hline Cluster & $\begin{array}{c}R_{\rho, \max } \\
(\mathrm{kpc})\end{array}$ & $\begin{array}{c}\rho_{0} \\
\left(10^{-20} \mathrm{~g} / \mathrm{m}^{3}\right)\end{array}$ & $\begin{array}{c}r_{\mathrm{c}} \\
(\mathrm{kpc})\end{array}$ & $\beta$ \\
\hline NGC 533 & 160 & $1.85(0.77)$ & $7(2)$ & $0.46(0.01)$ \\
Virgo & 35 & $8.14(0.85)$ & $3(1)$ & $0.33(0.01)$ \\
A262 & 199 & $4.54(0.91)$ & $5(1)$ & $0.33(0.01)$ \\
Sérsic 159-3 & 499 & $4.36(0.06)$ & $35(1)$ & $0.59(0.01)$ \\
MKW 9 & 238 & $67.7(16.29)$ & $19(8)$ & $0.32(0.02)$ \\
2A 0335+096 & 309 & $8.24(0.49)$ & $20(2)$ & $0.53(0.01)$ \\
MKW 3s & 402 & $2.61(0.12)$ & $35(2)$ & $0.49(0.01)$ \\
A2052 & 319 & $4.02(0.36)$ & $19(2)$ & $0.45(0.02)$ \\
A4059 & 412 & $1.71(0.18)$ & $48(7)$ & $0.50(0.02)$ \\
Hydra A & 673 & $5.13(0.70)$ & $29(5)$ & $0.52(0.02)$ \\
A496 & 193 & $5.14(0.14)$ & $16(1)$ & $0.42(0.01)$ \\
A3112 & 645 & $4.15(0.35)$ & $41(4)$ & $0.55(0.02)$ \\
A1795 & 553 & $4.26(0.18)$ & $42(3)$ & $0.52(0.01)$ \\
A399 & 606 & $0.72(0.08)$ & $76(15)$ & $0.38(0.03)$ \\
Perseus & 229 & $7.27(0.52)$ & $28(3)$ & $0.53(0.02)$ \\
A1835 & 831 & $5.24(0.23)$ & $80(5)$ & $0.62(0.02)$ \\
\hline \multicolumn{5}{|c}{}
\end{tabular}

\section{Effervescent heating}

Assuming spherical symmetry, the thermodynamic equations describing the ICM can be written in the form:

$v \rho r^{2}=$ const. $=\frac{\dot{M}}{4 \pi}$

$M_{\mathrm{tot}}=-\frac{r^{2} v}{G} \frac{\mathrm{d} v}{\mathrm{~d} r}-\frac{k T r}{G \mu m_{\mathrm{p}}}\left[\frac{\mathrm{d} \ln T}{\mathrm{~d} \ln r}+\frac{\mathrm{d} \ln \rho}{\mathrm{d} \ln r}\right]$

$H=\varepsilon-\varepsilon_{\text {cond }}+\varepsilon^{\star}$

where

$\varepsilon^{\star}=\frac{\rho v k T}{\mu m_{\mathrm{p}} r}\left[\frac{3}{2} \frac{\mathrm{d} \ln T}{\mathrm{~d} \ln r}-\frac{\mathrm{d} \ln \rho}{\mathrm{d} \ln r}\right]$,

and $v$ is the gas flow velocity which is taken positive outwards and $\dot{M}$ is the mass flow rate, which is therefore negative for an inflow and assumed, throughout the paper, to be constant. Here, $M_{\text {tot }}$ is the total gravitational mass within the radius $r$ and $\rho$, $T$ are the gas density and temperature, respectively ( $\mu=0.61$ is the mean molecular weight). Further, $\varepsilon=n_{\mathrm{e}}^{2} \Lambda(T)$ is the plasma emissivity and $\varepsilon^{\star}$ is the energy due to the inflow/outflow of the gas. Then, $\varepsilon$ is computed from the deprojected electron density $n_{\mathrm{e}}$ and $\Lambda(T)$, the cooling function for a plasma with an average metallicity $Z=0.5 Z_{\odot}$ losing energy by bremsstrahlung and line emission. The quantity $H$ in the energy equation (Eq. (6)) is thus an extra heating term. In the framework of the effervescent heating model developed in RB02, the extra heating is provided by buoyant bubbles injected into the ICM by the central AGN (see this section below).

The total gravitational mass $M_{\text {tot }}$ given in Eq. (5) differs from the mass estimated under the assumption of hydrostatic equilibrium, since it includes a velocity term. For the values of $\dot{M}$ considered in Sect. 4 below, this term is negligible, so the mass analysis presented in Piffaretti et al. (2005) gives accurate values for the mass profiles of the clusters in the present work.

For all the clusters in our sample, the entropy of the gas increases monotonically moving outwards, almost proportional to the radius (Piffaretti et al. 2005). Given this evidence, the convection term in the original effervescent heating model is neglected, since a declining entropy profile is essential for convection to operate (see the discussion in Ghizzardi et al. 2004). 
The term $\varepsilon_{\text {cond }}$ is the heating due to thermal conduction, which is given by

$\varepsilon_{\text {cond }}=\frac{1}{r^{2}} \frac{\mathrm{d}}{\mathrm{d} r}\left(r^{2} \kappa \frac{\mathrm{d} T}{\mathrm{~d} r}\right)$,

where $\kappa$ is the conductivity. For an ionized plasma, $\kappa$ is the Spitzer conductivity:

$\kappa=\kappa_{\mathrm{S}}=\frac{1.84 \times 10^{-5}(T)^{5 / 2}}{\ln \Lambda} \operatorname{erg~cm}^{-1} \mathrm{~s}^{-1} \mathrm{~K}^{-1}$,

where $\ln \Lambda \sim 40$ is the Coulomb logarithm.

Magnetic fields at the $\mu \mathrm{G}$ level are known to be present in clusters of galaxies, while higher values, up to tens of $\mu \mathrm{G}$, have been measured at the center of CF clusters (see Govoni \& Feretti 2004 , for a recent review). In the presence of such fields thermal conduction is suppressed below the Spitzer rate given in Eq. (9) by a factor $\sim 100-1000$ (Binney \& Cowie 1981; Chandran \& Cowley 1998). This condition has been essential for the development of multiphase CF models (e.g., Fabian 1994), but recent work has shown that the level of suppression of thermal conduction might not be as high as previously thought. In particular, Narayan \& Medvedev (2001) have shown that if the magnetic field is chaotic over a wide range of length scales, thermal conduction is enhanced to $\sim 1 / 5$ times the Spitzer value. Gruzinov (2002) pointed out that the effective heat conduction in a random variable magnetic field is boosted to a factor of 3 below the Spitzer value. The latter result yields the upper limit for the efficiency of heat conduction in the ICM. Therefore, in our analysis presented in Sect. 4, we investigate models with thermal conduction varying from zero to the maximum level of $1 / 3$ times the Spitzer value. The ICM conductivity $\kappa$ is therefore characterized by the fraction $f_{\mathrm{c}}$ according to $\kappa=f_{\mathrm{c}} \times \kappa_{\mathrm{S}}$.

Since thermal conduction is most efficient at high temperatures and it generally fails to balance radiative losses in the central parts of CF clusters, a heat source able to supply energy in the central parts is needed. Such a central heat source is incorporated in the RB02 model, in which AGNs are assumed to inject buoyant bubbles into the ICM, which heat the ambient medium by doing $p \mathrm{~d} V$ work as they rise and expand adiabatically. Here we summarize the derivation of the AGN heating function $H^{\mathrm{AGN}}$ (Begelman 2001). Assuming a steady state, the energy flux avaliable for heating is:

$\dot{e} \propto p_{\mathrm{b}}^{\left(\gamma_{\mathrm{b}}-1\right) / \gamma_{\mathrm{b}}}$,

where $p_{\mathrm{b}}$ is the pressure of the bubbles and $\gamma_{\mathrm{b}}$ their adiabatic index. Assuming that the bubbles' partial pressure scales as the ICM pressure $p$, the heating function $H^{\mathrm{AGN}}$ can be expressed according to:

$H^{\mathrm{AGN}}=-h(r)\left(\frac{p}{p_{0}}\right)^{\left(\gamma_{b}-1\right) / \gamma_{b}} \frac{1}{r} \frac{\mathrm{d} \ln p}{\mathrm{~d} \ln r}$,

with

$h(r)=\frac{L}{4 \pi r^{2}}\left(1-\mathrm{e}^{-r / r_{0}}\right) q^{-1}$

and where

$q=\int_{0}^{+\infty}\left(\frac{p}{p_{0}}\right)^{\left(\gamma_{\mathrm{b}}-1\right) / \gamma_{\mathrm{b}}} \frac{1}{r} \frac{\mathrm{d} \ln p}{\mathrm{~d} \ln r}\left(1-\mathrm{e}^{-r / r_{0}}\right) \mathrm{d} r$.

Here, $p_{0}$ is some reference ICM pressure (in the following we take its value at the cluster center) and $L$ the time-averaged luminosity of the central source. The term $1-\exp \left(-r / r_{0}\right)$ introduces an inner cutoff that fixes the scale radius where the bubbles start rising in the ICM. Therefore an inner cutoff is already taken into account when performing the integration in Eq. (13) and with zero as the lower integration limit gives a correct estimate for the time-averaged luminosity of the AGN. It must be noted, however, that the upper integration limit in Eq. (13) should be replaced by a finite number, i.e. the radius within which the bubbles effectively deposit heat into the ICM. This issue is explored in Sect. 4.5 since it is through Eq. (13) that the time-averaged luminosity of the central source is estimated and a finite integration limit has the effect of diminishing its energy requirement.

Finally, we notice that the following results are not only valid for the AGN-heating mechanism just described (i.e., heating by buoyant bubbles), but for any mechanism for which its dependence on the ICM pressure is equivalent to the one given in Eq. (11).

\section{Results}

From the observed deprojected gas temperature and density profiles, the required extra heating $H$ can be computed for fixed values of the mass deposition rate $\dot{M}$ and the conduction efficiency $f_{\mathrm{c}}$, and then fitted using the AGN heating function $H^{\mathrm{AGN}}$. For each bin with measured gas density and temperature, we compute the gas emissivity $\varepsilon$. For a fixed pair $\left(\dot{M}, f_{\mathrm{c}}\right)$, the conductive heat $\varepsilon_{\text {cond }}$ and the heat term $\varepsilon^{\star}$ are computed using the temperature and gas parametrizations given in Eqs. (1) and (3), since they depend on the gradients of these quantities. Then $\varepsilon_{\text {cond }}$ and $\varepsilon^{\star}$ are evaluated at the radius where the gas emissivity is computed, to finally obtain extra heating $H$ through Eq. (6).

The errors of $H$ are computed from the errors $(1 \sigma)$ of the gas emissivity and the $\varepsilon_{\text {cond }}$ and $\varepsilon^{\star}$ errors. The latter have been evaluated by propagating the $1 \sigma$ errors of the gas density and temperature best-fit parameters. While two different radial ranges have been used to fit the gas density and temperature profiles $\left(R_{\rho, \max }\right.$ and $R_{\mathrm{T}, \max }$, see Sect. 2.1), the extra heating $H$ is computed in bins within $R_{\mathrm{T}, \max }$, since $R_{\mathrm{T}, \max }<R_{\rho, \max }$. Finally the AGN heating function given in Eq. (11) is fitted to the extra heating data points using a $\chi^{2}$ minimization. The gas pressure and pressure gradients in Eq. (11) are evaluated using Eqs. (1) and (3). For any fixed pair $\left(\dot{M}, f_{\mathrm{c}}\right)$ the free parameters are therefore $r_{0}$ and the luminosity of the central AGN $L$. The bubbles' adiabatic index $\gamma_{\mathrm{b}}$ is fixed to $4 / 3$ throughout the paper (i.e., we assume relativistic bubbles), since the inclusion of $\gamma_{\mathrm{b}}$ as an additional free parameter leads to very large errors in the derived best-fit parameters. A discussion of the effects due to the use of different gas density and temperature parametrizations is given in Sect. 4.4.

\subsection{Zero AGN heating}

Despite the stability issues mentioned above, the case of thermal conduction by electrons acting as the only heat source has been already explored quite extensively in the literature, since it provides immediate information on the strength of heating by conduction in different clusters. Starting from Eqs. (4)-(6) one can estimate whether heat conduction alone can be efficient in quenching the cooling flow by setting both $H$ and $\dot{M}$ equal to zero. For a sample that includes all the objects studied in the present work, K04 have explored this possibility and concluded, in agreement with other studies (Voigt et al. 2002; Voigt \& Fabian 2004), that heat conduction alone is insufficient to balance radiative losses in CF clusters (with the exception of MKW 9 and A399, see also discussion below), in particular in their central regions. These results and the growing observational evidence of AGN-ICM interaction at the center of 
CF clusters motivate including in the model an additional heating term provided by a central AGN.

\subsection{Conduction and AGN heating: zero mass-dropout}

Motivated by the results of RB02, who find that the effervescent heating model does not require any mass dropout, we first present results for $\dot{M}=0$. As this case is the most justified, we present its predictions in more detail. In addition, as we show below, small mass-inflow/outflow rates do not modify the results substantially. For $\dot{M}=0$, we vary $f_{\text {c }}$ between 0 and $1 / 3$ and fit the extra heating curve $H$ (Eq. (6)) using the heating function $H^{\mathrm{AGN}}$ (Eq. (11)). No constraints are imposed on the AGN luminosity $L$, but the best-fit values for $r_{0}$ are searched in the interval from 0 to the cooling radius $R_{\text {cool }}$, which is computed for a cooling time of $15 \mathrm{Gyr}$ and is listed in Table 3.

In Fig. 1 we illustrate results for the cluster A1795 and discuss them in the following, since the model outcome for this object highlights important features also found for most of the clusters in the sample. Since no mass dropout is present, $\varepsilon^{\star}$ is zero in Eq. (6) and the extra heating $H=\varepsilon-\varepsilon_{\text {cond }}$ only depends on the conduction efficiency $f_{\mathrm{c}}$. Since $f_{\mathrm{c}}=1 / 3$ is the maximum value we consider, it corresponds to the maximum energy yield by heat conduction from the outer parts of the cluster. From a visual inspection of Fig. 1, it is clear that heat conduction is not able to lower the extra heating in the outermost bins. As a consequence, if the extra heating curve is fitted with Eq. (11), the resulting best-fit parameter $r_{0}$ (the scale radius where the bubbles start rising in the ICM) is unphysically large $\left(r_{0}=176 \mathrm{kpc}>R_{\mathrm{cool}}\right.$, see Table 3$)$ and the time-averaged AGN luminosity $L=3.3 \times 10^{45} \mathrm{erg} \mathrm{s}^{-1}$ is also quite large. The latter solution is not taken into account since, as stated above, the best-fit value for $r_{0}$ is searched in the interval from 0 to $R_{\text {cool }}$, but is used here as an example.

On the other hand, if the effervescent heating is assumed to be efficient in the region only within the cooling radius, one can notice that heat conduction is effective in reducing the extra heating so that the resulting extra heating curve is monotonically falling with radius. In this case one therefore expects that the extra heating supplied by the raising relativistic bubbles must be distributed over smaller distances and that the total AGN energy output is lower. This is indeed reflected in the much different best-fit parameters $r_{0}=14 \mathrm{kpc}$ and $L=8 \times 10^{44} \mathrm{erg} \mathrm{s}^{-1}$. This feature indicates that, as expected, the effervescent heating model strongly depends on how much and at which radial distance heat conduction is efficient. Because of this, we performed, for each cluster and each analytic function used to model the gas density and temperature, fits using the whole observed radial range (i.e., $0<r<R_{\mathrm{T}, \max }$ ) and the radial range delimited by the cooling radius.

Another common feature is the effect of the variation of the conduction efficiency. If $f_{\mathrm{c}}=0$ the extra heating curve is simply equal to the gas emissivity and the increase in $f_{\mathrm{c}}$ from 0 to $1 / 3$ gives a decrease in the extra heating curve from the emissivity curve to the data points marked in Fig. 1 . The increase in $f_{\mathrm{c}}$ should hence lead to a decrease in the AGN energy requirement. In fact we find that, within the cooling radius, both timeaveraged AGN luminosity and inner cutoff radius $r_{0}$ decrease monotonically with increasing $f_{\mathrm{c}}$. This opposite effect is seen if the fits are performed over the whole radial range: an increase in $f_{\mathrm{c}}$ leads to an increase in both $L$ and $r_{0}$, again showing the inadequacy of applying the model over the whole radial range.

For the majority of the objects in our sample, we find that the effervescent heating model provides results when the radial

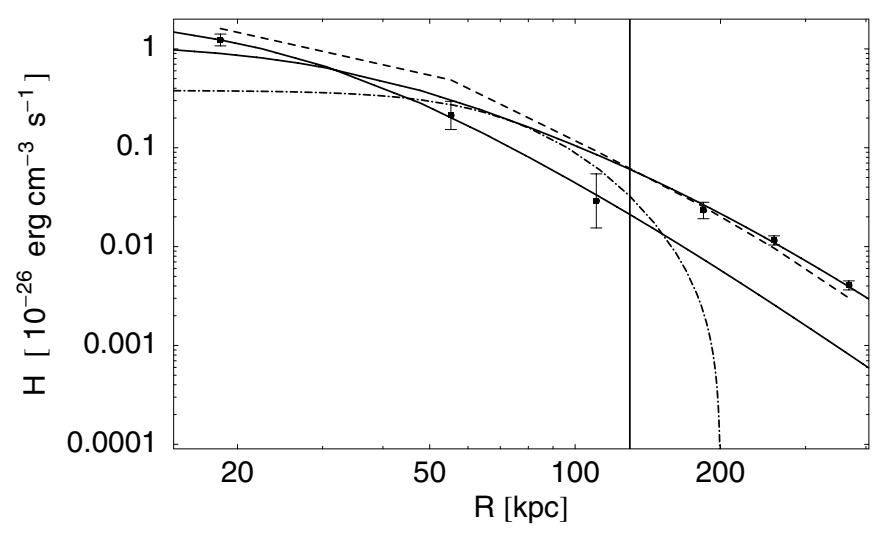

Fig. 1. Energy requirements in A1795. The plasma emissivity is shown, for simplicity, by the dashed straight lines joining the emissivity values computed in bins and heating due to thermal conduction by the dotdashed line. The mass flow rate is set to zero in this model, so the extra heating curve (filled squares) is determined by $f_{\mathrm{c}} \times$ Spitzer. Here $f_{\mathrm{c}}=$ $1 / 3$, the maximum value allowed in the models. The three bins inside the cooling radius (vertical line) alone are best fitted by an inner cutoff radius $r_{0}=14 \mathrm{kpc}$ and time-averaged AGN luminosity $L=8.0 \times$ $10^{44} \mathrm{erg} \mathrm{s}^{-1}$. For the same model $\left(\dot{M}=0\right.$ and $\left.f_{\mathrm{c}}=1 / 3\right)$, all six bins are best fitted by an unphysical $r_{0}=179 \mathrm{kpc}$ and very high AGN luminosity $L=3.3 \times 10^{45} \mathrm{erg} \mathrm{s}^{-1}$. The two solid curves are the best-fit functions for the two cases. See text for details.

range used to fit the extra heating curve with Eq. (11) is not simply the whole observed range but the radial range inside the cooling region. In fact for NGC 533, Virgo, A262, MKW 9, A4059, and A496, only fits performed over the radial range inside the cooling region converge. The reason for this lack of convergence of the fits is, as in the case of A1795 illustrated in Fig. 1, that heat conduction is not able to reduce the extra heating in the outermost bins. On the other hand, a reduction of the extra heating is present in bins close to the cooling radius.

This is a welcome feature for the effervescent heating model, because it implies that heating from the central source is not only more viable in terms of total energy output, but also that the energy can be distributed from the center outwards up to relatively small distances. For Sérsic 159-3, 2A 0335+096, Hydra A, and A1835 heat conduction is low even for the maximal conduction efficiency $f_{\mathrm{c}}=1 / 3$. Therefore a characteristic drop of the extra heating close to the cooling radius is not present in these objects and hence no difference is seen between the best fits performed using different radial ranges. The lowering of the extra heating close to the cooling radius is also not seen in MKW 3s, A2052, and $\mathrm{A} 3112$, because heat conduction is quite efficient only at the cluster center. While in A3112 heat conduction is low and therefore the best-fit parameters for the two radial ranges are almost identical, for MKW 3s and A2052 we obtain slightly higher values for both $r_{0}$ and $L$ when the whole radial range is used instead of the cooling region.

For these reasons, only results from fits to the extra heating curves within the cooling radius will be discussed. For A399, no results are obtained for either of the two radial ranges. The reason for the lack of convergence of the fits is that in A399 heat conduction is very efficient, owing to its relatively high temperature and very steep temperature profile (see also K04, Table 11). Conduction can balance radiative losses completely for $f_{\mathrm{c}}$ as low as 0.03 . For lower values of the conduction efficiency the best-fit values for $r_{0}$ are always larger than the cooling radius $R_{\text {cool }}=43 \mathrm{kpc}$ and therefore not taken into account. 
Table 3. Cooling radius $R_{\text {cool }}$ and the results for the effervescent heating model with no mass dropout $(\dot{M}=0)$. For each model, i.e. for a fixed conduction efficiency $0<f_{\mathrm{c}}<1 / 3$, the extra heating curve $H$ derived from Eqs. (4)-(6) is fitted using Eq. (11) over the radial range $0<r<R_{\text {cool }}$, and the best-fit values for $L$ and $r_{0}$ are derived. Only models with $f_{\mathrm{c}}$ between $f_{\mathrm{c}}^{\min }$ and $f_{\mathrm{c}}^{\max }$ can be fitted. The corresponding minimum and maximum values of the time-averaged luminosity $L$ and the inner cutoff radius $r_{0}$ are given. For most of the clusters, both best-fit parameters for time-averaged luminosity and inner cutoff radius decrease monotonically with increasing $f_{\mathrm{c}}$. These are labeled by an asterisk in the column trend. $\tilde{L}$ and $\tilde{r}_{0}$ are the time-averaged luminosity and the inner cutoff radius for the model with the smallest reduced $\chi^{2}$ and $\tilde{f}_{\mathrm{c}}$ is the corresponding conduction efficiency. The minimum and maximum values of the time-averaged luminosity $L_{\mathrm{fin}}^{\min \text { max }}$ for finite integration limits (see Sect. 4.5) are also listed. No result is found for A399.

\begin{tabular}{|c|c|c|c|c|c|c|c|c|c|c|c|}
\hline Cluster & $\begin{array}{l}R_{\text {cool }} \\
(\mathrm{kpc})\end{array}$ & $f_{\mathrm{c}}^{\min }$ & $f_{\mathrm{c}}^{\max }$ & $\begin{array}{c}r_{0}^{\min } \\
(\mathrm{kpc})\end{array}$ & $\begin{array}{l}r_{0}^{\max } \\
(\mathrm{kpc})\end{array}$ & $\begin{array}{c}\left(L^{\min }, L_{\text {fin }}^{\min }\right) \\
\left(\mathrm{erg} \mathrm{s}^{-1}\right)\end{array}$ & $\begin{array}{c}\left(L^{\max }, L_{\text {fin }}^{\max }\right) \\
\left(\mathrm{erg} \mathrm{s}^{-1}\right)\end{array}$ & trend & $\begin{array}{c}\tilde{r}_{0} \\
(\mathrm{kpc})\end{array}$ & $\begin{array}{c}\tilde{L} \\
\left(\operatorname{erg~s}^{-1}\right)\end{array}$ & $\tilde{f_{\mathrm{c}}}$ \\
\hline NGC 533 & 36 & 0.00 & 0.26 & 0.3 & 1.5 & $(1.6,0.5) \times 10^{42}$ & $(2.6,0.8) \times 10^{42}$ & $*$ & 1.5 & $2.6 \times 10^{42}$ & 0.00 \\
\hline Virgo & 24 & 0.03 & 0.26 & 0.6 & 17.9 & $(3.8,1.5) \times 10^{42}$ & $(2.1,0.4) \times 10^{43}$ & $*$ & 0.9 & $4.0 \times 10^{42}$ & 0.24 \\
\hline A262 & 61 & 0.12 & 0.33 & 6.6 & 60.4 & $(8.1,2.5) \times 10^{42}$ & $(4.7,0.6) \times 10^{43}$ & * & 6.6 & $8.1 \times 10^{42}$ & 0.33 \\
\hline Sérsic 159-3 & 128 & 0.00 & 0.33 & 21.6 & 22.6 & $(5.8,2.2) \times 10^{44}$ & $(5.8,2.3) \times 10^{44}$ & $*$ & 22.6 & $5.8 \times 10^{44}$ & 0.00 \\
\hline MKW 9 & 74 & 0.08 & 0.10 & 14.9 & 14.9 & $(5.7,0.5) \times 10^{42}$ & $(6.5,0.6) \times 10^{42}$ & * & 14.9 & $5.7 \times 10^{42}$ & 0.10 \\
\hline 2A $0335+096$ & 121 & 0.00 & 0.33 & 14.6 & 16.1 & $(6.1,2.3) \times 10^{44}$ & $(6.8,2.6) \times 10^{44}$ & $*$ & 14.6 & $6.1 \times 10^{44}$ & 0.33 \\
\hline MKW 3s & 95 & 0.00 & 0.33 & 37.2 & 94.0 & $(43.3,0.2) \times 10^{43}$ & $(58.1,7.3) \times 10^{43}$ & & 65.9 & $5.0 \times 10^{44}$ & 0.23 \\
\hline A2052 & 114 & 0.00 & 0.33 & 6.6 & 21.1 & $(2.6,0.7) \times 10^{44}$ & $(3.4,0.8) \times 10^{44}$ & & 17.6 & $3.4 \times 10^{44}$ & 0.25 \\
\hline A4059 & 86 & 0.00 & 0.33 & 4.7 & 53.8 & $(7.4,1.5) \times 10^{43}$ & $(5.0,0.6) \times 10^{44}$ & $*$ & 4.7 & $7.4 \times 10^{43}$ & 0.33 \\
\hline Hydra A & 130 & 0.00 & 0.33 & 26.5 & 29.4 & $(7.0,2.6) \times 10^{44}$ & $(7.7,2.8) \times 10^{44}$ & $*$ & 26.5 & $7.0 \times 10^{44}$ & 0.33 \\
\hline A496 & 104 & 0.07 & 0.28 & 11.1 & 86.3 & $(1.2,0.4) \times 10^{44}$ & $(5.7,0.8) \times 10^{44}$ & $*$ & 86.3 & $5.7 \times 10^{44}$ & 0.07 \\
\hline A 3112 & 141 & 0.00 & 0.33 & 29.9 & 58.7 & $(1.3,0.3) \times 10^{45}$ & $(1.4,0.4) \times 10^{45}$ & & 58.7 & $1.4 \times 10^{45}$ & 0.33 \\
\hline A1795 & 130 & 0.00 & 0.33 & 13.5 & 48.5 & $(8.0,2.3) \times 10^{44}$ & $(2.4,0.5) \times 10^{45}$ & * & 19.2 & $1.0 \times 10^{45}$ & 0.28 \\
\hline A399 & 43 & - & - & - & - & - & - & - & - & - & \\
\hline Perseus & 128 & 0.01 & 0.18 & 40.0 & 73.4 & $(1.6,0.5) \times 10^{45}$ & $(2.5,0.6) \times 10^{45}$ & $*$ & 40.0 & $1.6 \times 10^{45}$ & 0.18 \\
\hline A1835 & 204 & 0.00 & 0.33 & 33.2 & 37.8 & $(1.0,0.3) \times 10^{46}$ & $(1.1,0.4) \times 10^{46}$ & $*$ & 33.2 & $1.0 \times 10^{46}$ & 0.33 \\
\hline
\end{tabular}

The results for the zero mass dropout models are summarized in Table 3 . For each value of $f_{\mathrm{c}}$ the $1 \sigma$ errors on the best-fit parameters are very small compared to the range spanned by the best-fit parameters for the different models and therefore are not reported. In addition, it is important to notice that when fits are restricted to the cooling region, the number of bins we are using in the fits is quite low ( 3 for 9 objects, 4 for 4 objects, and 5 for 3 objects), and although it is sufficient for our scope, it should be noticed that for more complex models (e.g., models with $\gamma_{b}$ as an additional free parameter) the number of bins within the cooling radius should be increased if possible. While for most of the clusters we find a solution for $L$ and $r_{0}$ for every value of $0<f_{\mathrm{c}}<1 / 3$, for some objects the fits converge only for models with $f_{\mathrm{c}}$ in a narrower interval $\left(f_{\mathrm{c}}^{\min }-f_{\mathrm{c}}^{\max }\right.$, see Table 3$)$. In particular, in clusters where conduction is high (MKW 9 and Perseus), solutions are found for conduction efficiencies substantially lower than the maximum allowed value $f_{\mathrm{c}}=1 / 3$. In Sérsic 159-3, 2A0335+096, Hydra A, A3112, and A1835, the conductivity yield is low and, as expected, different models (i.e., different values of $f_{\mathrm{c}}$ ) give almost identical results.

It is crucial to highlight the trend of the best-fit parameters of the different models with model parameter $f_{\mathrm{c}}$. For most of the clusters, the model with $f_{\mathrm{c}}=f_{\mathrm{c}}^{\text {max }}$ is the one where values for both $L$ and $r_{0}$ are lowest and for $f_{\mathrm{c}}=f_{\mathrm{c}}^{\text {min }}$ the highest ( $L^{\text {min,max }}$ and $r_{0}^{\min , \max }$ in Table 3 ). In addition $L$ and $r_{0}$ vary monotonically with $f_{\mathrm{c}}$ within these limits. The clusters that exhibit this trend are labeled by an asterisk in column trend of Table 3. For MKW 3s, A2052, and A3112 the trend just described is reversed. In these objects heat conduction lowers the extra heating curve especially in the cluster center. This implies more and more flattening of the extra heating curve at the center with increasing $f_{\mathrm{c}}$, which is finally reflected in the increase in both $r_{0}$ and $L$. In Table 3 we also list the best-fit parameters $\tilde{L}$ and $\tilde{r}_{0}$ for the best model $\left(\tilde{f}_{\mathrm{c}}\right)$, which is chosen by simply comparing the $\chi^{2}$ of the best fit for the different models.

\subsection{Conduction and AGN heating: models with mass dropout or outflow}

Although RB02's results indicate that an equilibrium state with no mass dropout is usually achieved, the effervescent heating model incorporates the possibility of gas inflow or outflow. It is therefore interesting to see how much our results are changed by including gas inflow/outflow (negative/positive mass flow rates), since this implies a positive/negative work (per unit volume) done by the system ( $\varepsilon^{\star}$ in Eq. (6)). It is expected that negative mass-flow rates reduce the energy required by the central source to quench cooling, but the question is how large this effect is.

We apply the effervescent heating model as done in Sect. 4.2, but we go on to explore models with $-\dot{M}^{\max } / 10<\dot{M}<$ $+\dot{M}^{\text {max }} / 10$. When possible, the maximum mass flow rate $\dot{M}^{\text {max }}$ is set equal to the maximum of the mass deposition rates given in Table 5 in Peterson et al. (2003). Otherwise $\dot{M}^{\text {max }}$ is set equal to the maximum value that, at $f_{\mathrm{c}}=0$, still gives positive values for $H$ inside the cooling radius. The values of the maximum mass flow rates $\dot{M}^{\text {max }}$ are listed in Table 4 . This choice of the upper and lower bounds for $\dot{M}$ is dictated by the fact that we find the effect of a fixed mass inflow/outflow rate for all the clusters to be drastically different from cluster to cluster. In addition, taking RB02's results into account, low mass flow rates should be present when an equilibrium state (which is assumed here) is achieved. The analysis of models with $-\dot{M}^{\max } / 10<\dot{M}<$ $+\dot{M}^{\max } / 10$ is therefore a good compromise. We do not present the results from fitting the models extensively. More important we find that, as expected, the effect introduced by mass inflow or outflow is to reduce the time-averaged luminosity $L$ for inflows and enhancing it for outflows. From the models we have studied and, of course, for those where the fits converge in the $L-r_{0}$ plane, we report minimum and maximum values for the time-averaged luminosity $L$ and the inner cutoff radius $r_{0}$ in Table 4. A comparison with the values for the model with no 
Table 4. Results for the effervescent heating model with mass dropout or outflow. The minimum and maximum values of the time-averaged luminosity $L$ and the inner cutoff radius $r_{0}$ are computed from the results of models with conduction efficiency $0<f_{\mathrm{c}}<1 / 3$ and $-\dot{M}^{\text {max }} / 10<$ $\dot{M}<+\dot{M}^{\max } / 10$. The minimum and maximum values of the time-averaged luminosity $L_{\mathrm{fin}}^{\min m a x}$ for finite integration limits (see Sect. 4.5$)$ are also listed. As for the $\dot{M}=0$ models, no result is found for A399.

\begin{tabular}{l|c|cccc}
\hline \hline Cluster & $\begin{array}{c}\dot{M}^{\max } \\
\left(M_{\odot} / \mathrm{yr}\right)\end{array}$ & $\begin{array}{c}r_{0}^{\min } \\
(\mathrm{kpc})\end{array}$ & $\begin{array}{c}r_{0}^{\max } \\
(\mathrm{kpc})\end{array}$ & $\begin{array}{c}\left(L^{\min }, L_{\mathrm{fin}}^{\min }\right) \\
\left(\mathrm{erg} \mathrm{s}^{-1}\right)\end{array}$ & $\begin{array}{c}\left(L^{\max }, L_{\mathrm{fin}}^{\max }\right) \\
\left(\mathrm{erg} \mathrm{s}^{-1}\right)\end{array}$ \\
\hline NGC 533 & 5 & 0.2 & 13.5 & $(1.4,0.3) \times 10^{42}$ & $(3.1,1.0) \times 10^{42}$ \\
Virgo & 6 & 0.2 & 31.4 & $(3.3,1.3) \times 10^{42}$ & $(3.4,0.5) \times 10^{43}$ \\
A262 & 10 & 5.4 & 60.5 & $(6.1,2.0) \times 10^{42}$ & $(5.4,0.7) \times 10^{43}$ \\
Sérsic 159-3 & 210 & 20.9 & 23.4 & $(5.5,2.1) \times 10^{44}$ & $(6.1,2.4) \times 10^{44}$ \\
MKW 9 & 11 & 10.9 & 38.5 & $(4.1,0.4) \times 10^{42}$ & $(16.2,0.9) \times 10^{42}$ \\
2A 0335+096 & 420 & 14.4 & 16.1 & $(5.2,2.0) \times 10^{44}$ & $(7.7,2.9) \times 10^{44}$ \\
MKW 3s & 45 & 35.3 & 94.4 & $(4.3,0.5) \times 10^{44}$ & $(5.8,0.6) \times 10^{44}$ \\
A2052 & 100 & 4.6 & 89.4 & $(2.5,0.6) \times 10^{44}$ & $(4.5,0.9) \times 10^{44}$ \\
A4059 & 100 & 2.6 & 67.3 & $(5.2,1.0) \times 10^{43}$ & $(5.1,0.7) \times 10^{44}$ \\
Hydra A & 180 & 26.0 & 30.1 & $(6.6,2.4) \times 10^{44}$ & $(8.1,2.9) \times 10^{44}$ \\
A496 & 120 & 10.0 & 89.3 & $(1.1,0.3) \times 10^{44}$ & $(6.9,0.7) \times 10^{44}$ \\
A3112 & 300 & 27.7 & 72.2 & $(1.3,0.3) \times 10^{45}$ & $(1.5,0.4) \times 10^{45}$ \\
A1795 & 380 & 12.8 & 52.5 & $(6.6,1.9) \times 10^{44}$ & $(2.5,0.5) \times 10^{45}$ \\
A399 & 50 & - & - & - & - \\
Perseus & 650 & 34.4 & 78.5 & $(1.5,0.4) \times 10^{45}$ & $(2.8,0.6) \times 10^{45}$ \\
A1835 & 5800 & 24.1 & 53.6 & $(6.5,2.2) \times 10^{45}$ & $(1.5,0.4) \times 10^{46}$ \\
\hline
\end{tabular}

mass dropout (Table 3) shows that the broadening of the range for the best-fit parameters is not large.

\subsection{Effects of the temperature and density modelling}

In order to investigate the effects introduced by the choice of fitting functions for the density and temperature profiles, we performed the same analysis presented up to here (i.e., using Eqs. (1) and (3)) but using the remaining possible combinations of parametrizations given in Sect. 2.1. We find that for a fixed model (i.e., for fixed $\dot{M}$ and $f_{\mathrm{c}}$ ) the use of different parametrizations introduces small differences in the estimated best-fit parameters. These are smallest for the models without mass dropout or outflow and in particular for those clusters in which heat conduction is inefficient. For Sérsic 159-3, 2A0335+096, Hydra A, and A1835, the difference in both $L$ and $r_{0}$ is less than $3 \%$ and for the rest of the clusters less than $10 \%$. The differences are largest when the double $\beta$-model and Eq. (2) are used to parametrize gas density and temperature for models with $\dot{M} \neq 0$. Also in this case, the difference is smallest for the clusters in which heat conduction is not efficient (typically less then $7 \%$ for both best-fit parameters $L$ and $r_{0}$ ). For the rest of the clusters, the difference can be as high as $18 \%$, but generally less than $14 \%$. Taking other parametrizations of the temperature profile like those used in Churazov et al. (2003) and Dennis \& Chandran (2005) leads to a change of at most $10 \%$ for both best-fit parameters $L$ and $r_{0}$ for a fixed model. Most important, the trends between the AGN parameters $L$ and $r_{0}$ and the model parameters $f_{\text {c }}$ and $\dot{M}$ described in Sects. 4.2 and 4.3 remain unchanged for every combination of gas density and temperature parametrizations used. We therefore conclude that the values given in Tables 3 and 4 give quite robust estimates for the time-averaged luminosity $L$ and the inner cutoff radius $r_{0}$.

\subsection{Effects of the integration limits}

Since most of the models in which radiative cooling is balanced by some heating source fail due to insufficient energy yield, it is important to investigate to what extent our values for the time-averaged luminosity of the central AGN are overestimated. When fitting the extra heating function (Eq. (11)) to the extra heating curve, one directly derives the best-fit values for $r_{0}$ and the normalization of the extra heating function, i.e. $L / 4 \pi q$. The time-averaged luminosity $L$ is then estimated using Eq. (13). The values of $L$ reported so far were computed by setting the integration limits as given in Eq. (13), but as already noted in Sect. 3, the upper integration limit should be replaced. As reported in Sect. 4.2, the effervescent heating model provides results for almost all the clusters when applied to the cooling region. As a consequence we set the upper integration limit in in Eq. (13) equal to the cooling radius $R_{\text {cool }}$.

The effect of this replacement is to reduce the time-averaged luminosity of the central AGN. The effect of this simple but effective correction is shown in Table 3, where we list the minimum and maximum time-averaged luminosities $L_{\text {fin }}^{\min }$ and $L_{\text {fin }}^{\max }$ computed using this finite integration limit for the models with no mass dropout. The same quantities for the model with mass dropout are listed in Table 4. Comparing these values with the ones computed in the previous sections shows that the reduction in the time-averaged luminosity can be almost one order of magnitude, hence a finite integration limit in Eq. (13) should be used when estimating the effervescent heating best-fit parameters.

\section{Discussion and conclusions}

We have used deprojected radial density and temperature profiles of a sample of 16 nearby CF clusters observed with XMM-Newton (Kaastra et al. 2004) to test whether the effervescent heating model (Ruszkowski \& Begelman 2002) can satisfactorily explain the structure of CF clusters. The effervescent heating model incorporates both heat conduction and AGN feedback as heating sources, which is of great interest because of the complementary nature of these two processes. For each cluster, we derived the required extra heating as a function of clustercentric distance for various values of the unknown parameters $\dot{M}$ (mass deposition rate) and $f_{\mathrm{c}}$ (conduction efficiency). We fitted the extra heating curve using the AGN-heating function proposed by Ruszkowski \& Begelman (2002) and derived the 
AGN parameters $L$ (the time-averaged luminosity) and $r_{0}$ (the scale radius where the bubbles start rising in the ICM).

For models without gas mass dropout or outflow $(\dot{M}=0)$, we find:

- for only one object (A399) we do not find any solution for the effervescent heating model because heat conduction is very efficient;

- for 4 clusters (Sérsic 159-3, 2A0335+096, Hydra A, and A1835) the conductivity yield is extremely low and, as expected, different models (i.e., different values of $f_{\mathrm{c}}$ ) give almost identical results;

- for 3 objects (MKW 3s, A2052, and A3112), we find that heat conduction plays an important role only at the cluster center and that, as a consequence, the trend between the fitted AGN parameters and conduction efficiency is not the one expected if conduction and AGN heating are assumed to cooperate;

- for the remaining 8 clusters (NGC 533, Virgo, A262, MKW 9, A4059, A496, A1795, and Perseus), conduction and AGN heating are found to be cooperating.

We have studied models with mild gas mass dropout of outflow $\left(-\dot{M}^{\max } / 10<\dot{M}<+\dot{M}^{\max } / 10\right.$, with $\dot{M}^{\text {max }}$ being the mass deposition rate required by the standard $C F$ model) and find that:

- the conclusions listed above for the case of no mass dropout/outflow are also valid in this case;

- as expected, the effect introduced by mass inflow or outflow is to reduce the required AGN heating for inflows and to enhance it for outflows;

- this implies that the ranges of allowed AGN parameters $L$ and $r_{0}$ is broader than in the case $\dot{M}=0$, but we show that the broadening is not large.

We find that our results are not sensitive to the choice of fitting functions used to model gas density and temperature profiles and that the time-averaged AGN luminosities required to balance radiative losses are substantially reduced if the fact that the AGN deposits energy within a finite volume is taken into account.

Since we do not find any solution with the effervescent heating model for only one object (A399), we conclude that the model provides a satisfactory explanation of the observed structure of CF clusters. As pointed out by Begelman (2004), AGN-heating is dominant in the final stable state of the cluster modelled by Ruszkowski \& Begelman (2002). Conductive heating dominates only at the beginning of the evolution and might be essential only for the stability of the model. Therefore, models with high heat conduction are not preferred.

On the other hand, since there is evidence that AGN heating alone is not able to quench CFs (Brighenti \& Mathews 2002; Zakamska \& Narayan 2003), it is fair to assume that thermal conduction, although operating at different rates from cluster to cluster, must play an important role as a heating mechanism. While this is found for 8 clusters in our sample (NGC 533, Virgo, A262, MKW 9, A4059, A496, A1795, and Perseus), we have shown that conductive heating is either completely unimportant in 4 clusters (Sérsic 159-3, 2A0335+096, Hydra A, and A1835), too high for one object (A399), or high enough to play an important role but peaked at the cluster center in 3 clusters (MKW 3s, A2052, and A3112). Therefore, if we assume that AGN and conduction heating must be cooperating effectively, the model does not provide a satisfactory explanation for half of the objects in the sample.

These findings prompt us to posit that, at least for these objects, the description of their thermal structure through a steady

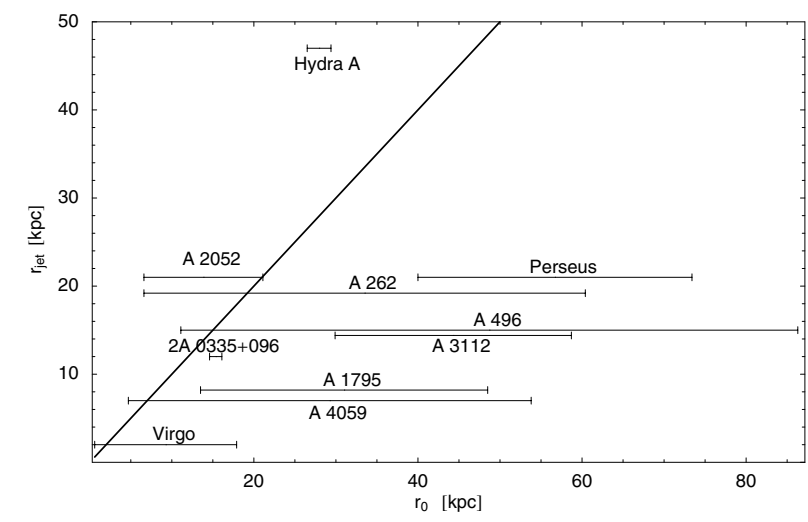

Fig. 2. Comparison between the observed extension of the AGN jet $r_{\text {jet }}$ and the best-fit AGN parameter $r_{0}$. For $r_{0}$ we plot the whole range of allowed values for the models with no mass dropout (see Table 3), and the extension of the AGN jet has been taken from the literature: Virgo (Young et al. 2002), A262 (Blanton et al. 2004), 2A 0335+096 (Sarazin et al. 1995), A2052 (Blanton et al. 2003), A4059 (Taylor et al. 1994), Hydra A (McNamara et al. 2000), A496 (Marković et al. 2004), A3112 (Takizawa et al. 2003), A1795 (Ettori et al. 2002), and Perseus (Fabian et al. 2002). The solid line indicates the locus of equality.

state solution of the thermodynamic equations is not viable and that we are observing them at an evolutionary stage far from equilibrium. A clearer picture can, of course, be achieved by studying a much larger sample using the procedure employed in this work.

For the clusters that are well described by the effervescent heating model, the derived best fit parameters $r_{0}$ and $L$ can be compared with results obtained from complementary observations. In the effervescent heating scenario, it is supposed that bubbles are deposited by the central radio source. Although the nature of this process is not well understood, an interesting possibility is that bubbles are generated through the interaction of the radio jets with the surrounding ICM. In the framework of the effervescent heating model, the AGN parameter $r_{0}$ fixes the scale radius where the bubbles start rising in the cluster atmosphere and heat the ICM. One then expects the AGN parameter $r_{0}$ to be larger than the jet extension and of the same order of magnitude. Since the observed jet extension $r_{\text {jet }}$ is at most as large as the true jet size due to projection effects, the same relationship is expected between $r_{0}$ and $r_{\text {jet }}$.

The size of the AGN jet has only been measured for 10 of the 15 clusters that are well described by the effervescent heating model. For these 10 objects, we show the comparison between $r_{0}$ and $r_{\text {jet }}$ in Fig. 2. Even though the range of allowed values for $r_{0}$ is quite large, the comparison shows that, for most of the clusters, the extension of the AGN jet and the scale radius $r_{0}$ are within the same order of magnitude and that the latter is in general larger than the jet extension. This implies that the possibility of bubbles being generated through the interaction of the radio jets with the ICM cannot be excluded.

A comparison between the derived luminosities $L$ with observed luminosities is by far more difficult. In fact, in the framework of the effervescent heating model, the derived AGN luminosity is a time-averaged total AGN power. Since the radio power is a poor tracer of the total power (Eilek 2004) a fair comparison is possible only for objects with estimated total jet power. At present, estimating the total jet power is unfortunately possible for only one source: M 87 in the Virgo cluster (Owen et al. 2000). In this case, our estimate of $L$ (see Table 3 ) agrees well with the inferred total jet power, 
$\sim 3-4 \times 10^{42} \mathrm{erg} \mathrm{s}^{-1}$. A similar conclusion is presented in Ghizzardi et al. (2004).

Even though we used the largest sample of CF clusters with well-measured deprojected gas density and temperature profiles available at present, it is clear that its size is not large enough to draw statistically significant conclusions on the viability of the effervescent heating model. Therefore, considering the simplicity of the procedure, any future work with the aim of extending the analysis to a much larger sample will provide vital information on this issue.

Acknowledgements. We wish to thank the referee for insightful comments that improved the presentation of the results. We also acknowledge Mateusz Ruszkowski for helpful remarks. This work is based on observations obtained with XMM-Newton, an ESA science mission with instruments and contributions directly funded by ESA Member States and the USA (NASA). RP acknowledges support from the Swiss National Science Foundation and the Tiroler Wissenschaftsfond. SRON is supported financially by the NWO, the Netherlands Foundation for Scientific Research.

\section{References}

Allen, S. W., Schmidt, R. W., \& Fabian, A. C. 2001, MNRAS, 328, L37

Ball, R., Burns, J. O., \& Loken, C. 1993, AJ, 105, 53

Begelman, M. C. 2001, Astron. Soc. Pac. Conf. Ser., 240, 363

Begelman, M. C. 2004, in Coevolution of Black Holes and Galaxies, from the Carnegie Observatories Centennial Symposia, ed. L. C. Ho (Cambridge University Press), 374

Binney, J., \& Cowie, L. L. 1981, ApJ, 247, 464

Bîrzan, L., Rafferty, D. A., McNamara, B. R., Wise, M. W., \& Nulsen, P. E. J. 2004, ApJ, 607, 800

Blanton, E. L., Sarazin, C. L., \& McNamara, B. R. 2003, ApJ, 585, 227

Blanton, E. L. 2004, The Riddle of Cooling Flows in Galaxies and Clusters of galaxies

Blanton, E. L., Sarazin, C. L., McNamara, B. R., \& Clarke, T. E. 2004, ApJ, 612, 817

Böhringer, H., Belsole, E., Kennea, J., et al. 2001, A\&A, 365, L181

Brighenti, F., \& Mathews, W. G. 2002, ApJ, 573, 542

Brüggen, M., \& Kaiser, C. R. 2001, MNRAS, 325, 676

Brüggen, M., \& Kaiser, C. R. 2002, Nature, 418, 301

Burns, J. O. 1990, AJ, 99, 14

Chandran, B. D. G., \& Cowley, S. C. 1998, Phys. Rev. Lett., 80, 3077
Churazov, E., Sunyaev, R., Forman, W., \& Böhringer, H. 2002, MNRAS, 332, 729

Churazov, E., Forman, W., Jones, C., \& Böhringer, H. 2003, ApJ, 590, 225

Colafrancesco, S., Dar, A., \& De Rújula, A. 2004, A\&A, 413, 441

David, L. P., Nulsen, P. E. J., McNamara, B. R., et al. 2001, ApJ, 557, 546

Dennis, T. J., \& Chandran, B. D. G. 2005, ApJ, 622, 205

Domainko, W., Gitti, M., Schindler, S., \& Kapferer, W. 2004, A\&A, 425, L21

Eilek, J. A. 2004, The Riddle of Cooling Flows in Galaxies and Clusters of galaxies [arXiv: astro-ph/0310011]

Ettori, S., Fabian, A. C., Allen, S. W., \& Johnstone, R. M. 2002, MNRAS, 331, 635

Fabian, A. C. 1994, ARA\&A, 32, 277

Fabian, A. C., Celotti, A., Blundell, K. M., Kassim, N. E., \& Perley, R. A. 2002, MNRAS, 331, 369

Fujita, Y., Matsumoto, T., \& Wada, K. 2004, ApJ, 612, L9

Ghizzardi, S., Molendi, S., Pizzolato, F., \& De Grandi, S. 2004, ApJ, 609, 638

Govoni, F., \& Feretti, L. 2004, Int. J. Mod. Phys. D, 13, 1549

Gruzinov, A. 2002 [arXiv: astro-ph/0203031]

Kaastra, J. S., Ferrigno, C., Tamura, T., et al. 2001, A\&A, 365, L99

Kaastra, J. S., Tamura, T., Peterson, J. R., et al. 2004, A\&A, 413, 415

Kaiser, C. R., \& Binney, J. 2003, MNRAS, 338, 837

Makishima, K., Ezawa, H., Fukuzawa, Y., et al. 2001, PASJ, 53, 401

Marković, T., Owen, F. N., \& Eilek, J. A. 2004, The Riddle of Cooling Flows in Galaxies and Clusters of galaxies

McNamara, B. R. 2004, The Riddle of Cooling Flows in Galaxies and Clusters of galaxies

McNamara, B. R., Wise, M., Nulsen, P. E. J., et al. 2000, ApJ, 534, L135

Narayan, R., \& Medvedev, M. V. 2001, ApJ, 562, L129

Owen, F. N., Eilek, J. A., \& Kassim, N. E. 2000, ApJ, 543, 611

Peterson, J. R., Paerels, F. B. S., Kaastra, J. S., et al. 2001, A\&A, 365, L104

Peterson, J. R., Kahn, S. M., Paerels, F. B. S., et al. 2003, ApJ, 590, 207

Piffaretti, R., Jetzer, Ph., Kaastra, J. S., \& Tamura, T. 2005, A\&A, 433, 101

Ruszkowski, M., \& Begelman, M. C. 2002, ApJ, 581, 223 (RB02)

Sarazin, C. L., Baum, S. A., \& O’Dea, C. P. 1995, ApJ, 451, 125

Soker, N. 2003, MNRAS, 342, 463

Soker, N., \& Sarazin, C. L. 1990, ApJ, 348, 73

Takizawa, M., Sarazin, C. L., Blanton, E. L., \& Taylor, G. B. 2003, ApJ, 595, 142

Tamura, T., Kaastra, J. S., Peterson, J. R., et al. 2001, A\&A, 365, L87

Taylor, G. B., Barton, E. J., \& Ge, J. 1994, AJ, 107, 1942

Voigt, L. M., Schmidt, R. W., Fabian, A. C., Allen, S. W., \& Johnstone, R. M. 2002, MNRAS, 335, L7

Voigt, L. M., \& Fabian, A. C. 2004, MNRAS, 347, 1130

Xue, Y., \& Wu, X. 2000, MNRAS, 318, 715

Young, A. J., Wilson, A. S., \& Mundell, C. G. 2002, ApJ, 579, 560

Zakamska, N. L., \& Narayan, R. 2003, ApJ, 582, 162 